Arq. Bras. Med. Vet. Zootec., v.66, n.6, p.1742-1750, 2014

\title{
Uso de plasma rico em plaquetas em úlceras de córnea em cães
}

\author{
[Use of platelet rich plasma on corneal ulcers in dogs]
}

\author{
N.B. Merlini ${ }^{1}$, J.F. Fonzar ${ }^{1}$, C.S. Perches ${ }^{1}$, M.G. Sereno ${ }^{1}$, V.L. Souza ${ }^{1}$, C.A. Estanislau ${ }^{1}$, \\ N.R. Rodas ${ }^{1}$, J J.T. Ranzani ${ }^{1}$, L. Maia ${ }^{1}$, C.R. Padovani ${ }^{2}$, C. V. S. Brandão ${ }^{1 *}$ \\ ${ }^{1}$ Faculdade de Medicina Veterinária e Zootecnia - Unesp - Botucatu, SP \\ ${ }^{2}$ Instituto de Biociências - Unesp - Botucatu, SP
}

\begin{abstract}
RESUMO
Este trabalho teve por objetivo avaliar, clínica e macroscopicamente, o tratamento adjuvante com plasma rico em plaquetas na forma de colírio ou tampão, em úlceras de córnea de cães atendidos no Serviço de Oftalmologia Veterinária. Foram analisados 20 olhos com diagnóstico de ceratite ulcerativa, distribuídos em dois grupos experimentais. O grupo colírio (GC) foi constituído por olhos tratados topicamente com colírio autólogo de plasma rico em plaquetas (PRP), e o grupo tampão (GT) por olhos submetidos ao tratamento à base de tampão sólido de PRP, associado ao recobrimento com terceira pálpebra para retenção deste. Os grupos foram avaliados, por meio de avaliação clínica, macroscópica e análise da redução do defeito epitelial, em diferentes momentos, aos três, cinco, dez, 15 e 30 dias, com exceção do terceiro dia no GT. O recobrimento da terceira pálpebra foi removido no quinto dia no GT. Em ambos os grupos, houve redução dos sinais de inflamação, melhora na sensibilidade ocular e adequada reparação do defeito epitelial. Todos os olhos do GT apresentaram completa cicatrização no quinto dia e 70\% no GC, atingindo a totalidade no $10^{\circ}$ dia. O PRP na forma de colírio ou tampão é uma excelente terapia adjuvante a ser instituída no tratamento clínico da úlcera de córnea em cães, pois atua na diminuição dos sinais inflamatórios, da dor ocular e auxilia potencialmente na cicatrização do defeito epitelial.
\end{abstract}

Palavras-chave: cão, defeito epitelial, fatores de crescimento, PRP

\begin{abstract}
This study aims to clinically and macroscopically evaluate the adjuvant therapy with platelet-rich plasma in the form of eyedrops or clot, for corneal ulcers in dogs treated at the Veterinary Ophthalmology Service. We analyzed 20 eyes diagnosed with ulcerative keratitis, divided into two experimental groups. The eyedrop group (GC) was composed of eyes treated topically with eyedrops of autologous plateletrich plasma (PRP), and the clot group (GT) was composed of eyes treated with a platelet-rich clot and covered with a third eyelid for retention of the clot. The groups were evaluated by clinical and macroscopic analysis and by the analysis of epithelial defect reduction, at different times, at three, five, ten, 15 and 30 days, except for the third day in GT. The coverage of the third eyelid was removed on the fifth day. In both groups the inflammation signs reduced, there was an improvement in ocular sensibility and proper repair of epithelial defect. All GT eyes and 70\% GC eyes showed complete healing on the fifth day, the remainder of GC completed healing on the tenth day. PRP in the form of eyedrops and clot is an excellent adjuvant therapy to be instituted in the clinical treatment for corneal ulcer in dogs, because it decreases the inflammatory signs and the ocular pain and it potentially assists in healing epithelial defects.
\end{abstract}

Keywords: dogs, epithelial defect, growth factors, PRP

Recebido em 2 de junho de 2013

Aceito em 10 de junho de 2014

*Autor para correspondência (corresponding author)

E-mail:valeriasb@fmvz.unesp.br 


\section{INTRODUÇÃO}

As lesões da córnea apresentam usualmente respostas patológicas associadas à perda de transparência, especialmente na ausência de terapia adequada. A úlcera de córnea é considerada uma emergência oftálmica, pois pode progredir para descemetocele ou perfuração ocular, com lesões, por vezes, irreversíveis e perda da visão (Gilger et al., 2007; Galera et al., 2009).

Os principais tratamentos clínicos adotados para úlcera de córnea referem-se à terapia antimicrobiana, à cicloplégica, aos analgésicos, aos agentes lubrificantes e aos fármacos antiproteases. Devido à alta incidência dessa afecção na clínica oftálmica, diversos são os estudos visando à reparação corneal, com o mínimo possível de sequelas, a fim de evitar a diminuição da acuidade visual (Gilger et al., 2007).

Os fatores de crescimento liberados pelos grânulos alfa das plaquetas são importantes no processo de regeneração tecidual, pois estimulam a angiogênese, regulam a inflamação (quimiotaxia) e a deposição da matriz extracelular (Anitua et al., 2004). O plasma rico em plaquetas (PRP) é uma concentração autóloga de plaquetas em um pequeno volume de plasma, obtido a partir da centrifugação do sangue total, com a consequente presença de fatores de crescimento liberados por essas plaquetas (Marx, 2004).

Vários fatores de crescimento presentes no PRP estão envolvidos na manutenção da estrutura e função corneal, especialmente os relacionados à transparência durante o processo de reparação (Imanishi et al., 2000). As plaquetas aderem-se ao tecido corneal lesado, liberando inúmeras citocinas e fatores de crescimento que induzem a mitose de fibroblastos, resultando na produção de colágeno (Alió et al., 2012).

Atualmente, na oftalmologia humana, o PRP vem sendo utilizado tanto na forma de colírio quanto de tampão sólido, bem como aplicação subconjuntival, para tratamento de defeito epitelial persistente, ceratoconjuntivite seca e úlcera de córnea por queimadura, e vem demonstrando excelente efeito, quanto à melhora nos sinais clínicos de inflamação, na acuidade visual dos pacientes e à cicatrização epitelial mais acentuada (Marquez et al., 2007; Alió et al., 2012; Kim et al., 2012; Panda et al., 2012).

Em oftalmologia veterinária, o uso do PRP na superfície ocular ainda não é muito difundido. Há na literatura apenas um estudo experimental em coelhos, no qual se preconizou o tratamento com colírio à base de PRP e tampão sólido de PRP associado à membrana aminiótica em úlcera de córnea induzida em coelhos, e o resultado foi que os animais tratados apresentaram menor opacidade corneal e cicatrização epitelial mais rápida em relação ao grupo controle (Donatti, 2010).

Nesse contexto, o objetivo do presente estudo foi avaliar, por meio de exames clínicos oftalmológicos, o uso do PRP sob a forma de colírio e tampão sólido associado ao recobrimento com terceira pálpebra, como adjuvante no tratamento de úlcera de córnea em cães.

\section{MATERIAL E MÉTODOS}

Foram utilizados 19 cães, machos e fêmeas, de diferentes raças, pesos e idade, perfazendo um total de 20 olhos, os quais foram distribuídos aleatoriamente em dois grupos experimentais com 10 olhos cada um. Os cães foram atendidos no setor de oftalmologia veterinária, com diagnóstico clínico de úlcera de córnea. Os critérios para inclusão no estudo compreenderam cães com úlceras superficiais, profundas e indolentes; obteve-se consentimento prévio dos proprietários para participação do estudo. O estudo foi submetido à Câmara de Ética em Experimentação Animal da Faculdade de Medicina Veterinária e Zootecnia-Unesp Campus Botucatu, protocolo número 142/2010CEUA.

Os animais foram submetidos a exame oftalmológico de rotina, com auxílio de uma lâmpada de fenda portátil (lâmpada de fenda portátil Kowa SL-15®), bem como ao teste lacrimal de Schirmer (teste de Schirmer Ophthalmos $\left.{ }^{\circledR}\right)$, à tonometria de aplanação (tonômetro de aplanação Tono Pen ${ }^{\circledR}$ - Med Tronic), ao teste de flurosceína (fluoresceína sódica $1 \%$ colírio - Allergan) e à oftalmoscopia direta (oftalmoscópio direto Welch Allyn ${ }^{\circledR}$ ) e fotodocumentação (câmera digital Nikon ${ }^{\circledR}$ 
Coolpix L1). Foram excluídos do estudo animais que apresentavam ceratoconjuntivite seca (KCS), bem como úlceras com profundidade superior a dois terços da espessura da córnea e descemetoceles. Alterações como cílios ectópicos e entrópio foram corrigidas ou removidas previamente.

Foram constituídos dois grupos experimentais, levando-se em consideração o tipo de tratamento empregado, sendo: grupo colírio (GC): olhos tratados topicamente com plasma rico em plaquetas, autólogo, na forma de colírio, administrado quatro vezes ao dia, por sete dias; grupo tampão (GT): olhos tratados por tampão sólido de plasma rico em plaquetas e recobrimento com a terceira pálpebra, com o objetivo de mantê-lo sobre a superfície ocular. Em todos os cães foi utilizada terapia antimicrobiana com colírio à base de tobramicina (Tobrex ${ }^{\circledR}-$ Alcon), quatro vezes ao dia, por sete dias.

A preparação do plasma rico em plaquetas seguiu rigorosa condição de esterilidade, com material estéril e descartável, e foi realizada dentro de uma capela de fluxo laminar unidirecional. Para o colírio de plasma rico em plaquetas, foram coletados $20 \mathrm{~mL}$ de sangue de cada um dos animais por punção da veia jugular, de maneira estéril. O volume total foi dividido em 10 frascos estéreis contendo citrato de sódio 3,2\% (tubo estéril com citrato de sódio 3,2\% Greiner Bio-one Brasil), resultando em dois mililitros $(\mathrm{mL})$ de sangue total em cada frasco.

A primeira etapa da centrifugação foi realizada a 220 gravidades $(\mathrm{g})$, durante 10 minutos, em centrífuga laboratorial comum (Sislab/Basic ${ }^{\circledR}$ ). A seguir, foram descartados aproximadamente $30 \%$ do plasma presente na superfície, com o propósito de usar um plasma mais concentrado em plaquetas na segunda centrifugação; o remanescente foi transferido para um tubo de Falcon seco e estéril. Em seguida, realizou-se a segunda etapa da centrifugação a 600 gravidades (g), por 10 minutos. Após a segunda centrifugação, o plasma foi dividido em duas frações: plasma pobre em plaquetas e plasma rico em plaquetas. Do sobrenadante, 2/3 foram descartados como plasma pobre em plaquetas (PPP) e 1/3 restante foi denominado plasma rico em plaquetas (PRP) e utilizado como colírio.
Este foi acondicionado a $4^{\circ} \mathrm{C}$ e utilizado durante uma semana.

Para a verificação do incremento plaquetário, foi utilizado o método de contagem plaquetária do PRP na forma de colírio em câmara de Neubauer, na diluição 1:100, sendo 20 microlitros do PRP em $2 \mathrm{~mL}$ de Brecher, resultando no número de plaquetas por microlitro.

Na preparação do tampão sólido de plasma rico em plaquetas, $10 \mathrm{~mL}$ de sangue foram coletados de cada animal por punção da veia jugular de maneira estéril, imediatamente antes da intervenção anestésica, finalizando seu preparo com a indução da coagulação no momento do procedimento cirúrgico. $\mathrm{O}$ processo de centrifugação se manteve o mesmo descrito para obtenção do colírio de PRP.

Após as duas etapas de centrifugação, o PRP foi transferido para o tubo tipo Eppendorf e adicionou-se cloreto de cálcio $10 \%$ ao PRP na proporção de 1:10, como descrito por Messora et al. (2007) e Alió et al. (2012); após a homogeneização, o tubo tipo Eppendorf foi colocado em banho-maria a $37^{\circ} \mathrm{C}$, durante dois minutos, para retração do coágulo e formação do tampão.

No grupo colírio, somente foi realizada a orientação quanto ao uso do colar elizabetano e do colírio tópico à base de plasma rico em plaquetas: lavar as mãos antes da aplicação, manter a área de aplicação limpa, evitar tocar o conta-gotas diretamente no olho do animal e manter em geladeira constantemente até o término do tratamento.

Os animais do grupo tampão foram submetidos ao recobrimento com a terceira pálpebra após a aplicação do tampão de PRP. Foram pré-medicados com acepromazina (Acepran ${ }^{\circledR}$ 0,2\% - Syntec) 0,03mg/kg e morfina (Dimorf® Cristália) 0,5mg/kg, ambos por via intramuscular, seguido por indução anestésica com propofol (Propovan ${ }^{\circledR}$ - Cristália) 5mg/kg, intravenoso, e manutenção com anestesia inalatória com isoflurano (Isoforine ${ }^{\circledR}$ Cristália), em sistema semifechado.

Os cães foram preparados de maneira rotineira, com prévia antissepsia, utilizando-se solução de 
polivinilpirrolidona (PVP - iodo antisséptico aquoso/Brasiliquidos Produtos Farmacêuticos/ Comercial Bono Ltda) a 1:50, sendo empregado pano fenestrado oftálmico estéril. Em seguida, aplicou-se o coágulo sólido de plasma rico em plaquetas, autólogo, na região da úlcera de córnea, procedendo-se, assim, ao recobrimento com terceira pálpebra. Nesse procedimento, institui-se a aplicação de sutura de colchoeiro, utilizando-se fio náilon monofilamentar 3-0, realizada entre as margens livres da terceira pálpebra e o aspecto lateral da pálpebra superior, com captons previamente confeccionados com sonda uretral 6, recobrindo, assim, toda a superfície corneal para garantir a manutenção do tampão de plaquetas sobre a lesão.

O primeiro momento de avaliação, designado M0 (momento inicial), deu-se na primeira consulta, quando foi confirmado o diagnóstico de úlcera de córnea. A seguir, os cães foram avaliados no M3 (três dias), M5 (cinco dias), M10 (10 dias), M15 (15 dias) e M30 (30 dias) do início do tratamento. O GT permaneceu com o recobrimento de terceira pálpebra durante cinco dias (M5), período a partir do qual foram iniciadas as avaliações pós-tratamento para o referido grupo, seguindo, então, os mesmos momentos descritos anteriormente.

Os sinais clínicos oftálmicos avaliados foram blefaroespasmo, fotofobia, hiperemia conjuntival, quemose, secreção ocular, opacidade de córnea e vascularização corneal. Esses foram qualificados e quantificados subjetivamente em (0) ausente; (1) leve; (2) moderado; (3) severo. Também foi aferida a espessura corneal previamente ao início do tratamento (M0) com o PRP e nos seguintes momentos de avaliação, M5, M15 e M30, para ambos os grupos experimentais, sendo realizadas três aferições na área da lesão e outras três na área adjacente lateral corneal com características clínicas oftalmológicas normais. Para sua realização, a córnea era dessensibilizada com o colírio à base de cloridrato de proximetacaína 0,5\% (Anestalcon ${ }^{\circledR}$ - cloridrato de proximetacaína 0,5\% - Alcon), seguindo-se a mensuração da espessura corneal por meio de delicado toque na córnea com a probe do paquímetro (paquímetro portátil SP-100 - Tomey - Japan). Foi estudada a porcentagem de variação da espessura corneal em relação ao momento basal.
A análise percentual da redução do defeito epitelial foi realizada após a instilação do colírio à base de fluoresceína (fluoresceína sódica 1\% colírio - Allergan), nos momentos de avaliação (M0, M3, M5, M10, M15 e M30), sendo realizada fotodocumentação com câmera digital (Nikon ${ }^{\circledR}$ Coolpix L1) e análise com o software Image $\AA$, programa gratuito desenvolvido pelo National Institute of Health (NIH).

Para as variáveis clínicas oftalmológicas, utilizou-se a análise de variância não paramétrica para o modelo de medidas repetidas em grupos independentes, complementada com o teste de comparações múltiplas de Dunn. O teste não paramétrico de Mann-Whitney foi utilizado para avaliação da porcentagem de redução da lesão (Zar, 2009). Para a porcentagem de positividade do teste de fluoresceína, foi utilizado o teste de Goodman para contrastes entre e dentro de proporções binominais (Goodman, 1964; Godman, 1965). Na espessura corneal, foi aplicada a técnica da análise de variância para o modelo de medidas repetidas, complementada com o teste de comparações múltiplas de Bonfennoni. Utilizou-se o nível de 5\% de significância (Zar, 2009).

\section{RESULTADOS E DISCUSSÃO}

O processamento do PRP foi realizado em câmara de fluxo laminar, o sangue foi coletado seguindo os preceitos assépticos, em tubos de citrato de sódio como anticoagulante, similar ao descrito por Alió et al. (2012), considerando-se que o uso do EDTA como anticoagulante não é recomendado para a preparação do PRP, pois este promove a fragmentação das plaquetas (Sanchez et al., 2003).

O acondicionamento do colírio de PRP à temperatura de $4^{\circ} \mathrm{C}$, durante uma semana, conserva todas as propriedades das plaquetas, como descrito por Alió et al. (2012) e Panda et al. (2012). A ativação das plaquetas acontece quando o colírio é instilado, pois, em contato com a superfície ocular, elas liberam os fatores de crescimentos de forma mais lenta, proporcionando um efeito prolongado (Alió et al., 2012).

O preparo do tampão sólido de PRP foi feito antes da indução anestésica do paciente e finalizado no momento do procedimento 
cirúrgico, induzindo sua coagulação, pois as plaquetas começam a liberar ativamente os fatores de crescimento cerca de 10 minutos após a coagulação. Trata-se de um procedimento importante na aplicação do tampão a ser seguido, uma vez que $95 \%$ dos fatores de crescimentos são secretados dentro de uma hora; após essa explosão inicial, as plaquetas continuam a sintetizar e a liberar proteínas e fatores de crescimento adicionais no período útil de sua vida (Eppley et al., 2006).

A concentração de plaquetas no sangue total estava dentro dos níveis fisiológicos preconizados para cães; de acordo com Meyer e Harvey (2004), 160.000 a 430.000 plaquetas/ $\mu \mathrm{L}$, portanto nenhum dos animais apresentou trombocitopenia. Segundo Barbosa et al. (2006), este seria um fator limitante para obtenção de concentrações adequadas de plaquetas no PRP, ou seja, quanto maior o número inicial de plaquetas, mais rico será o PRP.

O protocolo utilizado mostrou-se eficiente, pois alcançou média de 1.416 .242 plaquetas $/ \mu \mathrm{L}$ no PRP, com incremento plaquetário médio de quatro vezes no GC, e no GT média de 1.110.439 plaquetas $/ \mu \mathrm{L}$ no PRP, com incremento plaquetário médio de 3,96 vezes. Optou-se por centrifugação dupla, pois Marx et al. (1998) afirmam que o protocolo de dupla centrifugação é necessário para a concentração das plaquetas durante o preparo de PRP.

Segundo Marx (2004), para melhor eficiência do PRP, deve-se obter um aumento basal de plaquetas de quatro vezes a quantidade presente no sangue periférico ou uma concentração mínima de 500.000 a 1.000 .000 plaquetas $/ \mu \mathrm{L}$ para acelerar a reparação. Verificou-se, neste estudo, que tais parâmetros foram atingidos. Entretanto, Anitua et al. (2004) consideram que o valor superior a 300.000 plaquetas/ $\mu L$ já é suficiente para obtenção do PRP. Estudos mais recentes na oftalmologia, empregando o PRP na superfície ocular, como o de Alió et al. (2012), fazem referência à boa resposta terapêutica com concentrações de 1,6-2,5 vezes a quantidade basal de plaquetas para o colírio e de duas a três vezes para o tampão sólido, com única centrifugação. Kim et al. (2012), empregando concentração de 1,5 vez mais plaquetas, obtiveram uma dosagem alta de EGF e TGF- $\beta$, bem como boa resposta terapêutica.
Não houve diferença significativa entre os grupos, nos diferentes momentos, para as variáveis: secreção ocular, opacidade de córnea, vascularização corneal, fotofobia, hiperemia conjuntival e quemose. A diferença ocorreu apenas entre os momentos de cada grupo.

Quanto ao blefaroespasmo (Tab. 1), o grupo tampão apresentou maior intensidade dessa variável em comparação com o grupo colírio no M5. Acredita-se que esse comportamento tem uma relação direta com o momento da retirada do recobrimento com a terceira pálpebra. Na comparação entre os momentos, o grupo colírio apresentou redução significativa do blefaroespasmo do M0 para o M5, e o grupo tampão do M5 para o M10. Quanto à fotofobia, na comparação entre momentos, os grupos apresentaram redução significativa do M0 para o M5, permanecendo ausente até o final da avaliação. A fotofobia e o blefaroespasmo são sinais relacionados à dor, sendo duas das primeiras manifestações quando a superfície ocular é afetada; o processo de cicatrização promove diminuição do estímulo das terminações nervosas do epitélio e do estroma, promovendo, assim, a diminuição gradual dos sinais, conforme a processo de cicatrização avança (Andrade et al., 2009; Ferreira, 2012). De acordo com Alió et al. (2007) e (2012), o plasma rico em plaquetas, em úlceras indolentes em humanos, diminui os sinais relacionados à dor ocular, assim como sinais de inflamação.

Quanto à secreção ocular, cujo aspecto macroscópico variou de seroso a mucoso, verificou-se redução significativa, em relação ao momento inicial (M0), no M5 para o grupo colírio, com tendência a esta no grupo tampão.

No que diz respeito à opacidade corneal, ambos o grupos apresentaram escore leve na maioria dos momentos, sem diferenças no GT. No GC, entretanto, verificou-se melhora significativa do M15 para o M30. Salienta-se que alguns animais, no momento final de avaliação (M30), já não apresentavam opacidade corneal, especialmente no GC. Quanto à vascularização corneal entre grupos e momentos não foi observada diferença significativa. Processo de reparação rápido foi verificado, porémsendo que essa característica não foi ressaltada no estudo, embora seja um fator importante a ser considerado em casos mais refratários a tratamentos. 
Uso de plasma rico...

Tabela 1. Mediana e valores mínimos e máximos das variáveis blefaroespasmo e hiperemia conjuntival segundo grupo e momentos de avaliação (M) em dias

\begin{tabular}{|c|c|c|c|c|c|c|c|}
\hline Variável & Grupo & MO & M5 & M10 & M15 & M30 & Valor $\mathrm{p}$ \\
\hline \multirow[t]{2}{*}{ Blefaroespasmo } & Colírio & $2(1 ; 3) \mathrm{B}$ & $0(0 ; 1) \mathrm{A}$ & $0(0 ; 0) A$ & $0(0 ; 0) \mathrm{A}$ & $0(0 ; 0) A$ & $\mathrm{P}<0,05$ \\
\hline & Tampão & $3(1 ; 3) \mathrm{B}$ & $1(0 ; 1) \mathrm{AB}$ & $0(0 ; 0) A$ & $0(0 ; 0) A$ & $0(0 ; 0) \mathrm{A}$ & $\mathrm{P}<0,05$ \\
\hline Valor p & & $\mathrm{P}>0,05$ & $\mathrm{P}<0,05$ & $\mathrm{P}>0,05$ & $\mathrm{P}>0,05$ & $\mathrm{P}>0,05$ & \\
\hline Variável & Grupo & MO & M5 & M10 & M15 & M30 & Valor $\mathrm{p}$ \\
\hline \multirow{2}{*}{$\begin{array}{l}\text { Hiperemia } \\
\text { conjuntival }\end{array}$} & Colírio & $3(2 ; 3) \mathrm{B}$ & $1(0 ; 3) \mathrm{AB}$ & $1(0 ; 2) A$ & $0(0 ; 2) \mathrm{A}$ & $0(0 ; 1) \mathrm{A}$ & $\mathrm{P}<0,05$ \\
\hline & Tampão & $2(1 ; 3) C$ & $1(0 ; 2) \mathrm{BC}$ & $1(0 ; 1) \mathrm{AB}$ & $0(0 ; 1) \mathrm{A}$ & $0(0 ; 0) \mathrm{A}$ & $\mathrm{P}<0,05$ \\
\hline Valor p & & $\mathrm{P}>0,05$ & $\mathrm{P}>0,05$ & $\mathrm{P}>0,05$ & $\mathrm{P}>0,05$ & $\mathrm{P}>0,05$ & \\
\hline
\end{tabular}

Escores: 0-ausente; 1-leve; 2-moderado; 3-severo. * Duas medianas seguidas de pelo menos uma mesma letra maiúscula não diferem entre si $(\mathrm{P}>0,05)$.

Em relação à hiperemia conjuntival (Tab. 1), na comparação entre momentos, no GC, houve redução significativa dessa variável a partir do M10, bem como ausência nos momentos subsequentes de avaliação. No GT, essa variável também apresentou redução progressiva similar. Na quemose, verificou-se melhora significativa do M0 para o M5 no GC, e ausência nos demais momentos desse grupo e no GT. A melhora na inflamação deve-se aos fatores de crescimento liberados pelas plaquetas, que, além de estimularem a deposição da matriz extracelular, regulam a inflamação (quimiotaxia) (Anitua et al., 2004; Kim et al., 2012), fato esse também ressaltado por Alió et al. (2007), que descrevem a diminuição da inflamação após o uso de colírio à base de PRP em úlceras indolentes em humanos.
Na comparação da variação relativa da espessura em relação ao momento inicial (úlcera), notou-se que no M5 houve um aumento relativo significativo de $56 \%$ da espessura corneal em relação ao momento inicial no grupo colírio e de 5,7\% no grupo tampão (Tab. 2). Esse aumento na espessura pode ser decorrente da ainda presença de lesão epitelial no GC, pois, segundo Samuelson (2007), a remoção do epitélio produz um aumento de $200 \%$ da espessura em 24 horas, devido ao afluxo de água para o estroma corneal. O GT apresentava completa epitelização no teste de fluoresceína no M5, o que demonstra integridade epitelial e avanço no processo de reorganização celular, indicando que esse grupo provavelmente estava em fase de reparação mais adiantada que o GC no M5 (Pontes, 2010). Na periferia da córnea, não houve diferenças entre os grupos para a variável.

Tabela 2. Mediana e valores mínimo e máximo da porcentagem de variação da espessura corneal em relação ao momento basal, na região da úlcera, segundo grupo e momento de avaliação

\begin{tabular}{lccccc}
\hline Variável & Grupo & M5 & M15 & M30 & Valor p \\
\cline { 3 - 6 } Centro & \multirow{5}{*}{ colírio } & $(-26,67 ; 65,37)$ & $(-29,53 ; 90,32)$ & $(-33,35 ; 74,08)$ & $\mathrm{P}>0,05$ \\
\cline { 2 - 6 } & \multirow{2}{*}{ tampão } & 5,72 & & & \\
& & $(-25,30 ; 237,18)$ & $(-36,15 ; 360,89)$ & $(-23,86 ; 295,48)$ & \\
\hline \multicolumn{2}{c}{ Valor p } & $\mathrm{P}<0,05$ & $\mathrm{P}>0,05$ & $\mathrm{P}>0,05$ & \\
\hline
\end{tabular}

No teste de fluoresceína, observou-se, em $40 \%$ dos olhos do GC, resultado negativo no M3; no M5, 30\% dos olhos do grupo colírio apresentavam úlcera de córnea, e no M10 todos os olhos foram negativos nesse grupo. No GT, todos os olhos apresentaram-se negativos para o teste de fluoresceína no M5, sendo notada 
melhor resposta ao teste $(\mathrm{P}<0,05)$ do GT frente ao GC no M5 (Tab. 3).

Considerando-se a avaliação da área pelo software (ImageJ ${ }^{\circledR}$ ), constataram-se $95,3 \%$ de redução da área da lesão, após cinco dias de tratamento no GC, enquanto no GT a redução da lesão foi de $100 \%$ (Tab. 4). Nos demais momentos, os resultados foram semelhantes.

Tabela 3. Porcentagem de positividade para o teste de fluoresceína

\begin{tabular}{lccccccc}
\hline Variável & Grupo & MO & M5 & M10 & M15 & M30 & Valor p \\
\hline $\begin{array}{l}\text { Positividade } \\
\text { Fluoresceína (\%) }\end{array}$ & Colírio & $100,0 \% \mathrm{c}$ & $30,0 \%$ b & $0,0 \%$ a & $0,0 \%$ a & $0,0 \%$ a & P $<0,05$ \\
& Tampão & $100,0 \% \mathrm{~b}$ & $0,0 \%$ a & $0,0 \%$ a & $0,0 \%$ a & $0,0 \%$ a & P $<0,05$ \\
& & & & & & & \\
\hline Valor p & & $\mathrm{P}>0,05$ & $\mathrm{P}<0,05$ & $\mathrm{P}>0,05$ & $\mathrm{P}>0,05$ & $\mathrm{P}>0,05$ & \\
\hline
\end{tabular}

O processo de reepitelização foi constatado em um momento mais precoce no grupo tampão, já que todos os olhos apresentaram esse padrão no M5. A associação do recobrimento com terceira pálpebra no GT, cujo principal objetivo foi oferecer uma proteção mecânica ao tecido lesado e manter o tampão em contato com a superfície ocular, foi efetiva. Alió et al. (2007) e Donatti (2010) referem-se a uma atuação positiva similar do tampão de PRP à membrana amniótica, pois, quando o tampão sólido de PRP é utilizado, é necessário algum meio mecânico para mantê-lo em contato com a superfície ocular. Destaca-se, aqui, a facilidade do uso do recobrimento de terceira pálpebra para aplicação clínica do tampão sólido de PRP, com vantagens de ser um procedimento rápido, não necessitar de instrumentais e fios específicos, bem como procedimentos anestésicos mais elaborados, possibilitando a maior disponibilidade de utilização do tampão de PRP na oftalmologia veterinária.

Tabela 4. Porcentagem de redução da lesão, analisada pelo software Image ${ }^{\circledR}$, no quinto dia após o início dos tratamentos à base de PRP

\begin{tabular}{lccc}
\hline Grupo & Mínimo & Mediana & Máximo \\
\hline \multirow{2}{*}{ Colírio } & $95,3 \%$ & $100,0 \%$ & $100,0 \%$ \\
& & & \\
Tampão & $100,0 \%$ & $100,0 \%$ & $100,0 \%$ \\
\hline
\end{tabular}

O tratamento à base do colírio de PRP também foi bastante efetivo na cicatrização corneal, e acredita-se que tenha proporcionado liberação necessária dos fatores de crescimento para aceleração do processo de reparação, evidenciando, assim, que o PRP não demonstrou qualquer interferência negativa na migração de células epiteliais (Donatti, 2010). No PRP, as plaquetas permanecem intactas e, assim, podem aderir à superfície ocular, atuando de forma biológica e mecânica; também secretam os fatores de crescimento que atuam sobre a superfície ocular, acelerando a reparação epitelial, além de modular a inflamação local e a angiogênese (Alió et al., 2012).

Panda et al. (2012) e Kim et al. (2012) compararam o tratamento com o soro autólogo e o colírio de plasma rico em plaquetas, ambos como adjuvante na terapia para úlcera por queimaduras e para úlcera indolente em humanos, respectivamente. Panda et al. (2012) verificaram uma cicatrização epitelial mais rápida e com menor opacidade corneal. Kim et al. (2012) dosaram a quantidade de fatores de crescimento (EGF e TGF- $\beta$ ) em ambas as terapias, e encontraram maior concentração desses fatores no PRP em relação ao soro. Os autores concluíram que a elevada concentração contribuiu para uma recuperação mais acentuada do defeito epitelial e que o PRP apresenta efeitos mais favoráveis que o soro autólogo para reparação corneal. Assim, a principal diferença entre o soro autólogo e o PRP é a presença das plaquetas, que contribuem com a liberação dos fatores de crescimento em número maior que o soro e por um tempo de liberação maior, proporcionando, assim, uma cicatrização mais rápida (Alió et al., 2012; Panda et al., 2012; Kim et al., 2012).

Frente ao encontrado por Donatti (2010) em coelhos, o PRP apresenta uma atuação positiva à reparação corneal, indicando o seu uso para o tratamento de ceratite ulcerativa, pois é um 
material disponível, que apresenta atuação no local da lesão, sem efeitos sistêmicos adversos, além de ser de fácil aplicação no local desejado.

Assim, a terapia tópica com PRP é um complemento seguro e eficaz para o tratamento médico padrão em lesões da superfície ocular em humanos. Neste estudo, essa propriedade foi observada também em cães, indicando a sua aplicação clínica em doenças da córnea e também da superfície ocular.

Destaca-se, neste estudo, que, apesar de em alguns pontos o tampão de PRP apresentar características discretamente mais favoráveis, ambos foram clinicamente muito favoráveis e similares na maioria das análises realizadas; isto possibilita que a escolha do método a ser utilizado fique a critério do oftalmologista veterinário em cada caso específico. Ou seja, em animais cujo porte seja muito pequeno e/ou nos casos em que o proprietário tenha dificuldades na medicação tópica constante, a escolha pode ser pelo PRP na forma de tampão; em animais que, por motivos adversos, não possam ser submetidos a procedimentos anestésicos, opte-se pelo colírio.

O plasma rico em plaquetas é um material biológico disponível, de fácil processamento, necessitando de equipamentos básicos e metodologia adequada nas condições ideais de assepsia. O uso do PRP em oftalmologia vem se mostrando uma ferramenta eficaz na cicatrização de lesões da superfície ocular, pois proporciona uma alta concentração de fatores de crescimento que desempenham um papel fundamental no processo de cicatrização corneal.

\section{CONCLUSÕES}

De acordo com a metodologia instituída neste experimento, conclui-se que o plasma rico em plaquetas, na forma de colírio ou de tampão sólido, é um excelente adjuvante no tratamento de úlcera de córnea em cães, pois atua na diminuição dos sinais inflamatórios e da dor ocular, além de auxiliar potencialmente na cicatrização do defeito epitelial. Os protocolos de preparação adotados na confecção do plasma rico em plaquetas na forma de colírio e de tampão foram efetivos, bem como o recobrimento com a terceira pálpebra na manutenção do tampão de PRP.

\section{AGRADECIMENTOS}

À Fapesp (Fundação de Amparo à Pesquisa e Ensino do Estado de São Paulo), pelo apoio financeiro.

\section{REFERÊNCIAS}

ALIÓ, J.L.; ABAD, M.; ARTOLA, A. et al. Use of autologous platelet-rich plasma in the treatment of dormant corneal ulcer. Ophthalmology, v.114, p.1286-1293, 2007.

ALIÓ, J.L.; MONTIEL, F.A.; RODRIGUEZ, A.E. The role of "eye platelet rich plasma" (eprp) for wound healing in ophthalmology. Curr. Pharm. Biotechnol., v.13, p.1257-1265, 2012.

ANDRADE, A.L.; GOMES, J.A.P.; LUVIZOTTO, M.C.R. et al. Aspectos clínicos e morfológicos do transplante da membrana amniótica sobre a córnea de coelhos com deficiência induzida de células germinativas do limbo. Vet. Zootec., v.16, p.127-142, 2009.

ANITUA, E.; ANDIA, I.; ARDANZA, B. et al. Autologous platelets as a source of proteins for healing and tissue regeneration. Thromb. Haemost., v.91, p.4-15, 2004.

BARBOSA, A.L.T.; DEL CASTRO, R.J.; GOMES, B.N.; MONTEIRO, B.S. Plasma rico em plaquetas: uma fonte de múltiplos fatores de crescimento para enxertos ósseos. Arq. Bras. Med. Vet. Zootec., v.58, p.1-14, 2006.

DONATTI, C. Uso do plasma rico em plaquetas sob a forma de colírio ou tampão no reparo de úlceras de córnea profundas induzidas em coelhos: Avaliação clínica e histomorfométrica. 2010. 106f. Dissertação (Mestrado) - Faculdade de Medicina Veterinária e Zootecnia. Universidade Estadual Paulista, Botucatu.

EPPLEY, B.L.; PIETRZAK, W.S.; BLANTON, M. Platelet rich plasma: a review of biology and applications in plastic surgery. Plast. Reconstr. Surg., v.118, p.147-156, 2006.

FERREIRA, G.T.N.M. Aspectos clínicos do implante da membrana amniótica criopreservada em associação ou não com transplante de limbo $e$ do enxerto conjuntival $360^{\circ}$ no tratamento de úlceras de córnea em cães. 2012. 75f. Dissertação (Mestrado) - Faculdade de Medicina Veterinária, Universidade Estadual Paulista, Araçatuba. 
GALERA, P.D.; LAUS, J.L.; ORIÁ, A.P. Afecções da túnica fibrosa. In: LAUS, J.L. Oftalmologia Clínica e Cirúrgica em Cães e Gatos. São Paulo: Rocca Ltda, 2009. p.69-96.

GILGER, B.C.; BENTLEY, E.; OLLIVIER, F.J. Diseases and surgery of the canine cornea and sclera. In: GELATT, K.N. Veterinary Ophthalmology. 4.ed. Iowa: Blackwell Publishing, 2007. Chap.15, p.690-752.

GOODMAN, L.A. Simultaneus confidence intervals for contrast among multinomial populations. Ann. Math. Stat., v.35, p.716-725, 1964.

GOODMAN, L.A. Simultaneus confidence intervals for multinomial proportions . Technometrics, v.7, p.247-254, 1965.

IMANISHI, J.; KAMIYAMA, K.; IGUCHI, I. et al. Growth factors: importance in wound healing and maintenance of transparency of the cornea. Prog. Retin. Eye res., v.19, p.113-129, 2000.

KIM, K.M.; SHIN, Y.T.; KIM, H.K. Effect of autologous platelet rich plasma on persistent corneal epithelial defect after infectious keratitis. Jpn. J. Ophthalmol., v.56, p.544-550, 2012.

MARQUEZ, R.A.; ESPINOSA, I.M.; MUNOZ, M.; PEREIRA, G. Aplicación subconjuntival de concentrado de plaquetas plasmáticas en el tratamiento de quemaduras oculares. Resultados preliminares. Arch. Soc. Esp. Oftalmol., v.82, p.475- 482, 2007.

MARX, R.E. Platelet-rich plasma: evidence to support its use. J. Oral maxillofac. Surg., v.62, p.489-496, 2004.
MARX, R.E.; CARLSON, E.R.; EICHSTAEDT, R.M. et al. Platelet-rich plasma: growth factor enhancement for bone grafts. Oral surg. Oral med. Oral pathol. Oral radiol. Endod., v.85, p.638-646, 1998.

MESSORA M.R.; NAGATA, M.J.H.; MARIANO, R.C. et al. Bone healing in criticalsize defects treated with platelet-rich plasma: a histologic and histometric study in a rat calvaria. J. Periodontal. Res., v.10, p.1-7, 2007.

MEYER, D.J.; HARVEY, J.W. Veterinary laboratory medicine: interpretation e diagnosis. 2.ed. Philadelphia: Saunders, 2004. 351p.

PANDA, A.; JAIN, M.; VANATHI, M. et al. Topical Autologous Platelet rich plasma eyedrops for acute corneal chemical injury. Cornea, v.31, p.989-993, 2012.

PONTES, K.C.S. Membrana amniótica xenógena, associada ou não ao $\mathrm{N}$-butil 2cianoacrilato, no tratamento de lesão corneal perfurada. Estudo experimental em coelhos. 2010. 93f. Tese (Doutorado). Universidade Federal de Viçosa, Viçosa.

SANCHEZ, A.R.; SHERIDAN, P.J.; KUPP, L.I. Is Platelet-rich plasma the perfect enhancement factor? A Current review. Int. J. Oral Max. Impl., v.18, p.93-103, 2003.

SAMUELSON, D.A. Ophtalmic Anatomy. In: GELATT, K.N. Veterinary Ophtalmology. 4.ed. Iowa: Blackwell Publishing, 2007. p.37-148.

ZAR, J.H. Biostatistical Analysis. 5.ed. New Jersey: Prentice-Hall, 2009. 960p. 